\title{
Escala de Parentalidad Percibida (EPP): medir psicométricamente en las configuraciones familiares actuales
}

\author{
Guadalupe de la Iglesia ${ }^{1}$
}

\begin{abstract}
RESUMEN
El objetivo de esta investigación fue estudiar psicométricamente una medida de parentalidad percibida adaptable a las configuraciones familiares actuales y a los objetivos de investigación vigentes en el área. Para ello se analizó una muestra de adolescentes $\left(n=443, M_{\mathrm{EDAD}}=15.49\right.$ años, $D E_{E D A D}=1.85,50.6 \%$ varones, $49.4 \%$ mujeres) y una muestra de adultos $\left(n=512, M_{\mathrm{EDAD}}=40.83\right.$ años, $D E_{E D A D}=15.05,49.6 \%$ varones, $50.4 \%$ mujeres) utilizando un instrumento diseñado especialmente para este fin. Los análisis efectuados derivaron en un instrumento (Escala de Parentalidad Percibida; EPP) de veinte elementos pasible de ser utilizado tanto en adolescentes (EPP-ADO) como en adultos (EPP-ADU) con familias de origen diversas. La medida cuenta con evidencias de validez que fundamentan su estructura bidimensional en todas sus versiones y que habilitan a estudios comparativos de los resultados obtenidos con las mismas. Además, los análisis con distintos criterios externos replican los resultados comúnmente hallados cuando se analiza la parentalidad percibida en relación con la sintomatología psicológica, el bienestar y los estilos de afrontamiento. Se proveen baremos para su transferencia al ámbito de aplicación. Mediante la EPP se pueden estudiar las dos dimensiones centrales de la parentalidad (respuesta y demanda) y su combinación en el análisis de estilos parentales tanto en cuidadores primarios como secundarios.
\end{abstract}

Palabras clave: parentalidad, estilos parentales, validez de constructo, invarianza factorial, adultos, adolescentes.

\section{Perceived Parenting Scale (PPS): psychometric measures for current family configurations}

\begin{abstract}
The main objective of this research was to analyze a psychometric measure of perceived parenting adaptable to current family configurations and research topics in the area. For that a sample of adolescents $\left(n=443, M_{\mathrm{AGE}}=15.49\right.$ years old, $S D_{A G E}=1.85,50.6 \%$ male, $49.4 \%$ female) and a sample of adults was studied $\left(n=512, M_{\mathrm{AGE}}=40.83\right.$ years old, $S D_{A G E}$ $=15.05,49.6 \%$ male, $50.4 \%$ female) using a measure specially designed to meet this end. The analyses concluded in a twenty-item measure (Perceived Parenting Scale; PPS) that can be used in adolescents (PPS-ADO) and adults (PPS-ADU) from diverse type of families. The measure entails validity evidences that give empirical support to its bidimensional structure in all of its versions and that enable comparative studies of the results obtained in any of them. Also, the analyses carried out with different external criteria replicate those commonly found when perceived parenting is studied in relation to psychological symptoms, well-being and coping styles. Punctuation scales are provided for the applied field. The PPS may be used to study the two main dimensions of parenting (responsiveness and demandingness) as well as their combination in parenting styles of primary and secondary caregivers.
\end{abstract}

1 Consejo Nacional de Investigaciones Cientificas y Técnicas (CONICET), Universidad de Palermo, Universidad de Buenos Aires, Argentina; gdelaiglesia@gmail.com; http://orcid.org/0000-0002-0420-492X 
Keywords: parenting, parenting styles, construct validity, factorial invariance, adults, adolescents.

En el siglo XX las prácticas de crianza dejaron de estar completamente fundamentadas en valores filosóficos, humanísticos o religiosos y emergió el estudio científico de la parentalidad que los sujetos experimentaron en su niñez y adolescencia (Baumrind, 1966; Maccoby, 1992). El interés central en esta área ha rondado en el análisis de los estilos de crianza y el grado de ajuste psicológico a lo largo de todo el ciclo vital. El mayor cúmulo de investigaciones ha estado focalizado en población infantil y adolescente, y en menor cantidad en población adulta, a pesar de que se ha señalado que la parentalidad vivenciada tiene efectos a lo largo de todo el ciclo vital (Anisman, Zaharia, Meaney, \& Merali, 1998; Aquilino \& Supple, 2001; Kasser, Koestner, \& Lekes, 2002; Luecken, Appelhans, Karft, \& Brown, 2006; Maccoby, 1994; Rohner \& Veneziano, 2001; Rothrauff, Cooney, \& An, 2009).

Debe recalcarse que en las investigaciones sobre parentalidad, en general se da mayor peso a la percepción que los sujetos tienen del comportamiento de los padres por sobre la conducta real de estos últimos (Ausubel et al., 1954; Sánchez Gutiérrez, 2009; Schaefer, 1965; Serot \& Teevan, 1961). Esto se denomina perspectiva cognitivista y se fundamenta en que los participantes de una interacción no reaccionan simplemente a las acciones del otro, sino que su comportamiento se relaciona con su propia interpretación de lo que el otro está haciendo (López, Palacio, \& Nieto, 2007; Maccoby \& Martin, 1983). De hecho, en una investigación en la que se compararon medidas de parentalidad de niños, sus madres y observadores, se halló una mayor y significativa correspondencia entre lo reportado por los observadores y los niños -más que la verificada entre observadores y madres- (Sessa, Avenevoli, Steinberg, \& Morris, 2001).

En cuanto al estudio de la parentalidad como constructo teórico, existe un amplio consenso en cuanto a las dimensiones centrales que componen la parentalidad: la respuesta parental y la demanda parental (e.g., Baumrind, 1996). Esta delimitación se puede visualizar incluso en teorias muy diversas (e.g., Erikson, 1963; Minuchin, 1974). La dimensión respuesta aborda el grado de afectividad expresado por los padres en términos de cariño, preocupación, contención, diálogo, cercanía, calidez, apoyo, escucha (Ainsworth, Bell, \& Stayton, 1971; Baumrind, 1991a, 1994, 1996; Maccoby \& Martin, 1983; Rohner, 2004). La dimensión demanda refiere a la puesta en marcha de estrategias para regular (controlar, manejar) los comportamientos de los hijos. Aquí se incluyen exigencias, limites, prohibiciones, castigos, reglas o normas, disciplina, firmeza. Estas dimensiones suelen estudiarse por separado y también combinadas mediante el análisis de estilos parentales. En este sentido, el aporte de Maccoby y Martin (1983) fue fundante de una categorización que se sigue utilizando hoy en dia de cuatro estilos parentales: autoritario, permisivo, negligente y autoritativo. En el estilo autoritario -alta demanda y baja respuesta- el énfasis está en el control, en la obtención de obediencia y en el uso del castigo para la regulación del comportamiento (Baumrind, 1966, 1991a, 1991b; Dwairy, 2008; Maccoby \& Martin, 1983). Los progenitores con estilo negligente -baja demanda y baja respuesta- no son punitivos ni tampoco responsivos, desatienden sus responsabilidades como 
padres (Baumrind, 1966, 1991a, 1991b). El estilo permisivo -baja demanda y alta respuesta- se caracterizan por la pretención de que los hijos autorregulen sus propias conductas y tomen sus propias decisiones, son padres de calidad y evitan la confrontación y el control de las conductas de sus hijos (Baumrind, 1991a, 1991b; Dwairy, 2008). El estilo autoritivo -alta demanda y alta respuesta- se caracteriza por la presencia tanto de calidez como de control con el fin de dar lugar a una gradual autonomía. Estos padres utilizan el razonamiento para impartir límites, justificando sus decisiones y reglas, dando lugar al diálogo y la escucha (Baumrind, 1996, 1991a, 1991b; Maccoby \& Martin, 1983).

Se ha analizado la adecuación y pertinencia del uso de estos dos abordajes en el estudio de la parentalidad -dimensional y categórico- (Darling \& Steinberg, 1993). Algunos autores defienden la evaluación dimensional fundamentando su elección en el hecho de que la dimensión de responsividad resulta -según el estado del arte- la más influyente, sumado al hecho de que la investigación mediante tipologías es en ocasiones dificultosa, ya que alegan que no es posible clasificar con exactitud los estilos parentales (Kim \& Rohner, 2002). Otros proponen como más productivo y abarcador un análisis desde categorias de estilos parentales, basándose en que sin dudas ambas dimensiones se influyen mutuamente y sería incorrecto considerar el efecto de una sin tener en cuenta el de la otra (Baumrind, 1975, 1991a; Maccoby \& Martin, 1983). Ante un fenómeno tan amplio y heterogéneo a lo largo del tiempo y de distintas culturas, puede localizarse un extenso espectro de trabajos encarados desde ambas perspectivas.

\section{Parentalidad y variables asociadas}

Existen distintas variables asociadas tanto a las dos dimensiones de la parentalidad como a los cuatro estilos del abordaje categorial. A modo de ejemplo, se sabe que, en población infantil, la dimensión respuesta está asociada al incremento en la cantidad de vocabulario, mejores habilidades sociales y menor agresividad, mayor autorregulación y mayor desarrollo prosocial y al mayor uso del afrontamiento aproximativo en niños (Farrant \& Zubrick, 2011; Gaylord-Harden, Campbell, \& Kesselring, 2010; Jennings et al. 2008; Kaiser, McBurnett, \& Pfiffner, 2011; Richaud de Minzi, 2010; Valiente, Lemery-Chalfant, \& Swanson, 2009). En adolescentes, se encuentra relacionada con un mayor ajuste psicológico, mayor autoestima y menos sintomas depresivos, menor consumo de alcohol y drogas, mayor satisfacción con la vida, mayor resolución de conflictos y menor agresividad hacia los pares (Behnke, Plunkett, Sands, \& Bámaca-Colbert, 2011; Brody et al., 2009; Clark, Belgrave, \& Abell, 2012; Dwairy \& Dor, 2009; Kokkinos \& Voulgaridou, 2016; Lee, 2011; Missotten, Luyckx, Van Leeuwen, Klimstra, \& Branje, 2016; Ozer, Flores, Tschann, \& Pasch, 2011; Richaud de Minzi, Lemos, \& Mesurado, 2011; Richaud de Minzi, Mesurado, \& Lemos, 2012; Rohner, 2010; Rohner, Khaleque, Elias \& Sultana, 2010; Schwarz et al., 2011); y menos conductas externalizantes y mayor estabilidad emocional, menos sintomas paranoides, mejor ajuste psicológico, menor ansiedad, menor uso de pensamiento dialéctico, menor cantidad de signos de trastornos de personalidad y mayor uso de afrontamientos de tipo aproximativos en adultos (Chyung \& Lee, 2008; de la Iglesia, 2013; Khaleque \& Rohner, 2012; Mehrad Sadr, 2016; Riggio, 2004; Riggio \& Kwong, 2011; Ripoll-Nuñez \& Álvarez, 2008; Xu et al., 2016). 
La dimensión demanda, por el contrario, se halló asociada a mayores sentimientos de soledad, más neuroticismo y, en consecuencia, menos estado de flow, y al mayor uso de afrontamiento evitativo en niños (Caycedo et al., 2005; Gaylord-Harden et al., 2010; Mesurado \& Richaud de Minzi, 2012; Richaud de Minzi, 2006; Zhou et al., 2008); a más externalización de problemas, menor ajuste psicológico, menos conductas prosociales y menor agresividad hacia los pares en adolescentes (Benson, Buehler, \& Gerard, 2008; Dwairy \& Dor, 2009; Kokkinos \& Voulgaridou, 2016; Ozer et al., 2011; Richaud de Minzi et al., 2011); y a mayor presencia de conductas de desórdenes alimentarios, mayor uso de afrontamientos de tipo evitativo y mayor cantidad de signos de trastornos de personalidad en adultos (de la Iglesia, 2013; Reilly, Stey, \& Lapsley, 2016; Xu et al., 2016).

En cuanto a los estilos parentales, se sabe que el autoritario se asocia a baja autoestima en niños (Pinquart $\&$ Gerke, 2019), a menor ajuste psicológico en adolescentes (Dwairy, 2008) y a menor bienestar en adultos (Aguilar Villalobos, Martínez Jiménez, \& Valencia Cruz, 2003). El estilo negligente se relaciona con más emociones negativas y menor expresividad en niños (Lengua \& Kovacs, 2005; Muljat, Wilson, Oliver, Kottke, \& Petaja, 2008), con menor autoestima en adolescentes (Pinquart \& Gerke, 2019), y menor calidad de vínculos familiares y con seres significativos en la adultez (Aguilar Villalobos et al., 2003). El estilo permisivo se relaciona con la presencia de mayor autoestima en niños (Pinquart \& Gerke, 2019), mayor autoestima, ajuste psicológico y menos conductas problemáticas en adolescentes (García \& Gracia, 2009), y mayor bienestar y vida familiar, más comportamientos antisociales y menos apego seguro en adultos (Aguilar Villalobos et al., 2003; Schaffer, Clark, \& Jeglic, 2009; Sun, Ng, \& Guo, 2010). Por último, el estilo autoritativo se ha encontrado asociado a un aumento de las habilidad de regulación de la atención y la conducta en niños (Cheah, Leung, Tahseen, \& Schultz, 2009), menos comportamientos vinculados a la enfermedad y más comportamientos asociados a la salud, mayor autoestima, ajuste psicológico y menos conductas problemáticas, menos delincuencia y depresión, y menos problemas de conducta en adolescentes (García \& Gracia, 2009; Lohaus, Vierhaus, \& Ball, 2009; Saint-Jacques \& Lépine, 2009; Simons \& Conger, 2007) y a mayor bienestar, armonía familiar, autoestima, vida familiar y morosidad en adultos (Aguilar Villalobos et al., 2003).

Los antecedentes mencionados no fueron diferenciados por progenitor para no complejizar la redacción. De todos modos, la mayoría de las investigaciones demuestran mayores resultados asociados a la percepción de parentalidad ligada a la madre por sobre el padre (e.g., Xu et al., 2016).

\section{Nuevas formas familiares y medición psicométrica de la parentalidad}

Existen numerosos instrumentos diseñados para evaluar la parentalidad, tanto desde una perspectiva dimensional como categórica, y dirigidos a población infantil, adolescente y de adultos. Entre los más utilizados se encuentran el Children's Report of Parental Behavior (CRPB; Schaefer, 1965), Parental Acceptance- Rejection Questionnaire (PARQ; Rohner, Saavedra, \& Granum, 1978) y el Parental Bonding Inventory (Parker, Tupling, \& Brown, 1979). En general, todos ellos apuntan a la valoración de las prácticas parentales paternas, por un lado, y maternas, por el otro. En la mayoria se asume que el evaluado pertenece a una familia compuesta por un padre y una madre. Aunque el tipo de familia predominante es aún la nuclear, compuesta 
por padre, madre e hijos (del Campo, 2004), la versatilidad de la organización familiar a lo largo del tiempo y en el mundo (Ocón, 2006) se puede vislumbrar en la diversidad de configuraciones familiares existentes en la actualidad. Todas ellas se enmarcan en la denominación de nuevas formas familiares (Ruiz Becerril, 2004). Ninguno de los tests psicométricos para medir parentalidad cuenta con la versatilidad suficiente para adaptarse a las distintas configuraciones familiares vigentes hoy en dia.

Dado lo expuesto, se propone construir un test para la medición de la parentalidad percibida que resulte ser una medida con apropiadas propiedades psicométricas y de fácil administración que sea versátil para las distintas formas familiares. Además, que pueda ser utilizada tanto en población adolescente como adulta para la comparabilidad del fenómeno en esas poblaciones.

\section{MÉTODO}

\section{Participantes}

En este estudio se trabajó con dos muestras: una de adolescentes y otra de adultos. La muestra de adolescentes estaba compuesta por 443 adolescentes de población general argentina de edad promedio de 15.49 años $(D E=1.85)$ y estaban distribuidos equitativamente según sexo $(50.6 \%$ varones, $49.4 \%$ mujeres). Todos ellos se encontraban cursando la escuela secundaria. La mayoría de los evaluados convivía con ambos padres $(71.8 \%)$, el $22.8 \%$ dijo solo convivir con su madre, el $3.8 \%$ solo convivir con su padre y el $2.1 \%$ dijo estar a cargo de otro adulto (ej: abuelo/a, tío/a). En cuanto a su situación económica, el $45.5 \%$ dijo que en su hogar "El dinero cubre los gastos del mes con tranquilidad", el 39.4 dijo "Aunque cuesta, el dinero cubre los gastos del mes", el 10.9\% "El dinero nunca es una preocupación", el 3.8\% "Es muy dificil que el dinero alcance para todo el mes" y el $0.5 \%$ "El dinero nunca nos alcanza para cubrir los gastos". En relación con la muestra de adultos, estaba constituida por 512 adultos de población general argentina de edad promedio 40.83 años $(D E=15.05)$ y estaban distribuidos equitativamente según sexo (49.6\% varones, $50.4 \%$ mujeres). En cuanto a su estado civil, el $42.6 \%$ dijo estar solo (soltero, divorciado/separado, viudo) y el $57.4 \%$ dijo estar en pareja (de novio, unido, casado). En cuanto a su nivel de estudios, la mayoria (93.1\%) tenía título de escuela secundaria o superior. Finalmente, el $66.4 \%$ dijo pertenecer poseer un nivel socio-económico medio, el $19.7 \%$ mediobajo, el $10.9 \%$ medio-alto, el $2.3 \%$ bajo, y el $0.6 \%$ alto.

\section{Materiales}

Escala de Parentalidad Percibida (EPP). Esta es una medida de parentalidad percibida con una versión para adolescentes (EPP-ADO) y otra para adultos (EPP-ADU). La versión preliminar del test cuenta con 42 items ideados para la medición de las dos dimensiones centrales de la parentalidad: respuesta y demanda. Los items se contestan en una likert de grado de frecuencia $(0=$ nunca a $3=$ siempre $)$ y estaban redactados en presente para el caso de los adolescentes y en pasado para el caso de los adultos. Con el objetivo de contar con mayor versatilidad a la hora de incluir diversas configuraciones familiares, se solicita a los evaluados que respondan refiriéndose a quién está a cargo de su crianza, contando con la opción de contestar refiriéndose a uno o a dos adultos a cargo de la crianza. Con este 
diseño, el test permite valorar la parentalidad percibida con relación al adulto que está mayormente a cargo de la crianza y/o a otro adulto que está a cargo de la crianza en la misma proporción o inferior. En el caso de que se hayan valorado a dos adultos, se puede analizar a cada uno por separado o estudiar el promedio de ambas percepciones. La construcción de esta escala fue el objetivo principal de este estudio. En los apartados siguientes se describe el procedimiento para su diseño y los análisis psicométricos realizados sobre la medida.

Listado de Sintomas Breve - 50 (LSB-50; de Rivera \& Abuín, 2012). Este instrumento fue administrado únicamente en la muestra de adolescentes. Está compuesto por 50 ítems que evalúan diferentes sintomas psicológicos que se pueden valorar globalmente mediante un Índice Global de Severidad. Los items se responden mediante una escala likert de cinco posiciones que varia entre $0=$ nada a $4=$ mucho. Esta escala fue adaptada localmente (de la Iglesia, Fernández Liporace, \& Castro Solano, 2015; de la Iglesia, Castro Solano, \& Fernández Liporace, 2016) en dos estudios que incluyeron el estudio de la dimensionalidad del listado mediante análisis factoriales confirmatorios con pruebas de validación cruzada e invarianza factorial. El instrumento cuenta además con evidencias de validez externa mediante la correlación de un instrumento de diagnóstico de psicopatología, así como también se analizó su consistencia interna.

Mental Health Continuum- Short Form (MHC-SF; Keyes, 2005). Este instrumento fue administrado únicamente en la muestra de adultos. Se trata de un instrumento destinado a la medición del bienestar en sus aspectos emocionales, psicológicos y sociales. Para este estudio se utiliza únicamente la puntuación total como una medida bienestar. Cuenta con 14 items con una escala likert de 5 puntos, que va de 0 (nunca) a 5 (todos los dias). En la adaptación argentina (Lupano Perugini, de la Iglesia, Castro Solano, \& Keyes, 2017) se ha confirmado la estructura tridimensional de la escala y una buena validez convergente.

Inventario de Estilos de Afrontamiento para Adolescentes y Adultos (Moos, 1993). Este instrumento fue administrado tanto en la muestra de adolescentes como en la de adultos y brinda cuatro medidas de estrategias de afrontamiento del estrés: aproximativo cognitivo, aproximativo conductual, evitativo cognitivo y evitativo conductual. Está compuesto por 22 elementos que se responden con un formato likert de cuatro posiciones $0=$ nunca a $3=$ siempre que refieren a frecuencia de uso de la respuesta de afrontamiento. La adaptación local (Ongarato, de la Iglesia, Ongarato, \& Fernández Liporace, 2009; Rial Boubeta, de la Iglesia, Ongarato, \& Fernández Liporace, 2011) contó con estudios de componentes principales, análisis factorial confirmatorio con validación cruzada en invarianza factorial, además de estudios de consistencia interna y estabilidad de las puntuaciones.

\section{Procedimiento}

El estudio fue correlacional, instrumental y no experimental. Los datos fueron recolectados por un muestreo intencional de estudiantes avanzados de la carrera de Psicologia bajo la supervisión de un investigador experto. Se solicitó la firma de un consentimiento informado que en el caso de la muestra de adolescentes fue firmado por los padres o tutores. En él se explicaba el objetivo de la investigación, se establecía que la participación de su hijo/a era 
voluntaria y que podía desistir de participar en cualquier momento, y que los datos serian tratados de manera confidencial y anónima.

El diseño de la Escala de Parentalidad Percibida estuvo guiado por un criterio racional. La versión de 42 items fue obtenida mediante la generación de reactivos, de modo tal que abarcara el constructo y las dimensiones que se pretendian medir. Previo a la administración de la escala en la muestra de participantes, el instrumento fue sometido a un juicio experto en donde cuatro profesionales con conocimientos sobre psicometría y dinámicas familiares realizaron recomendaciones para mejorar la calidad de la medida.

Tabla 1.

Estadísticos descriptivos para las versiones de EPP-ADO.

\begin{tabular}{|c|c|c|c|c|c|c|c|c|c|c|c|c|}
\hline \multirow[b]{2}{*}{ EPP-ADO } & \multicolumn{4}{|c|}{ MADRE } & \multicolumn{4}{|c|}{ PADRE } & \multicolumn{4}{|c|}{ PROMEDIO } \\
\hline & $M(D E)$ & $A$ & $C$ & $\mathrm{IH}_{\mathrm{C}}$ & $M(D E)$ & $A$ & $C$ & $\mathrm{IH}_{\mathrm{C}}$ & $M(D E)$ & $A$ & $C$ & $\mathrm{IH}_{\mathrm{C}}$ \\
\hline 1 & $0.70)$ & -1.28 & 1.19 & .64 & $9(0.86)$ & -0.78 & -0.24 & .70 & $.33(0.69)$ & -0.87 & 0.23 & .71 \\
\hline & $2.24(0.72)$ & -0.79 & 0.61 & .46 & $2.18(0.83)$ & -0.84 & 0.15 & .51 & $2.20(0.63)$ & -0.75 & 0.94 & .49 \\
\hline Ítem 3 & $2.33(0.78)$ & -0.97 & 0.29 & .54 & $2.11(0.89)$ & -0.73 & -0.31 & .64 & $2.21(0.74)$ & -0.79 & 0.17 & .61 \\
\hline Ítem 4 & $2.22(0.87)$ & -1.01 & 0.35 & .61 & $1.92(0.95)$ & -0.57 & -0.56 & .68 & $2.06(0.79)$ & -0.70 & -0.06 & .66 \\
\hline & $0.26(0.62)$ & 2.67 & 6.99 & .22 & $0.29(0.65)$ & 2.39 & 5.38 & .14 & $0.27(0.53)$ & 2.26 & 5.21 & .15 \\
\hline & $2.13(0.73)$ & -0.59 & 0.22 & .55 & $1.95(0.84)$ & -0.52 & -0.25 & .57 & $2.03(0.67)$ & -0.40 & -0.06 & .59 \\
\hline & 1.09 & 0.49 & -0.82 & .47 & $1.04(1.00)$ & 0.56 & -0.81 & .45 & $1.07(0.89)$ & 0.43 & -0.77 & .45 \\
\hline & 82) & -1.21 & 0.85 & .36 & $2.16(0.95)$ & -0.96 & -0.05 & .42 & $2.25(0.79)$ & -1.02 & .54 & .41 \\
\hline & $2.48(0.70)$ & -1.30 & 1.38 & .67 & $2.26(0.82)$ & -0.90 & 0.10 & .70 & $2.36(0.65)$ & -0.88 & 0.38 & .72 \\
\hline Íte & 1.56( & -0.01 & -1.08 & .40 & $1.35(1.00)$ & 0.16 & -1.05 & .40 & $1.45(0.82)$ & -0.01 & -0.60 & .38 \\
\hline Íte & 2.39 & -1.38 & 1.15 & .38 & $2.05(1.01)$ & -0.72 & -0.66 & .44 & $2.22(0.79)$ & -0.95 & 0.38 & .41 \\
\hline & 2.26 & -0.82 & -0.05 & .55 & 1.61 & -0.09 & -1.19 & .56 & $1.93(0.77)$ & -0.32 & -0.45 & .58 \\
\hline & 2.05 & -0.66 & -0.43 & .55 & 1.91 & -0.50 & -0.78 & .56 & $1.98(0.82)$ & -0.56 & -0.29 & .55 \\
\hline 14 & $2.35(0.75)$ & -1.03 & 0.62 & .72 & $1.99(0.90)$ & -0.43 & -0.78 & .70 & $2.16(0.71)$ & -0.61 & -0.02 & .73 \\
\hline Íter & $2.11(1.00)$ & -0.75 & -0.65 & .41 & $1.61(1.08)$ & -0.13 & -1.27 & .48 & $1.86(0.90)$ & -0.47 & -0.67 & .47 \\
\hline Íter & $2.67(0.63)$ & -2.14 & 4.74 & .51 & $2.53(0.76)$ & -1.62 & 1.96 & .67 & $2.59(0.60)$ & -1.55 & 2.03 & .64 \\
\hline 17 & $0.22(0.63)$ & 3.18 & 9.98 & .27 & $0.24(0.61)$ & 2.83 & 7.74 & .21 & $0.23(0.52)$ & 2.59 & 7.12 & .24 \\
\hline 18 & $2.33(0.76)$ & -0.96 & 0.47 & .69 & $1.98(0.85)$ & -0.43 & -0.59 & .74 & $2.15(0.69)$ & -0.56 & -0.04 & .74 \\
\hline 19 & $1.87(0.99)$ & -0.41 & -0.91 & .46 & $1.41(1.05)$ & 0.12 & -1.18 & .60 & $1.64(0.90)$ & -0.16 & -0.87 & .54 \\
\hline 20 & $1.52(0.96)$ & 0.03 & -0.95 & .60 & $1.43(1.03)$ & 0.11 & -1.13 & .63 & $1.47(0.86)$ & -0.03 & -0.82 & .61 \\
\hline 21 & $2.36(0.82)$ & -1.15 & 0.55 & .64 & $1.99(0.98)$ & -0.59 & -0.73 & .69 & $2.17(0.77)$ & -0.75 & -0.05 & .69 \\
\hline 22 & $1.43(0.99)$ & 0.10 & -1.04 & .60 & $1.35(1.06)$ & 0.16 & -1.20 & .69 & $1.39(0.92)$ & 0.10 & -0.92 & .66 \\
\hline 23 & $2.41(0.83)$ & -1.38 & 1.18 & .61 & $2.25(0.87)$ & -1.00 & 0.19 & .65 & $2.33(0.76)$ & -1.11 & 0.64 & .66 \\
\hline 24 & $0.58(0.90)$ & 1.45 & 1.05 & .17 & $0.58(0.87)$ & 1.39 & 0.92 & .17 & $0.58(0.77)$ & 1.27 & 0.75 & .13 \\
\hline 25 & $1.20(1.05)$ & 0.34 & -1.12 & .45 & $0.77(0.90)$ & 0.96 & 0.00 & .35 & $0.98(0.86)$ & 0.53 & -0.58 & .44 \\
\hline 26 & $2.26(0.90)$ & -1.07 & 0.23 & .72 & $1.84(1.01)$ & -0.43 & -0.92 & .75 & $2.05(0.83)$ & -0.69 & -0.11 & .75 \\
\hline & & -1.14 & 0.7 & .76 & & -0.60 & & .78 & 4) & -0.70 & 1 & .79 \\
\hline & & 0.07 & -1.09 & .57 & & 0.24 & & 60 & $1.39(0.92)$ & 0.10 & -0.97 & .59 \\
\hline & 2.32 & -1.03 & 0. & .69 & 1.86 & -0.42 & -1.04 & .70 & $2.08(0.80)$ & -0.60 & -0.31 & .72 \\
\hline & 2.36 & -1.18 & 0.81 & .15 & 2.27 & -1.03 & 0.30 & .14 & $2.31(0.73)$ & -1.06 & 0.85 & .12 \\
\hline & 2.35 & -1.15 & 0.85 & .68 & 2.1 & -0.74 & -0.19 & .76 & $2.22(0.74)$ & -0.80 & 0.18 & .75 \\
\hline & 0.34 & 2.34 & 4.55 & .22 & 0.3 & 2.35 & 4.96 & .21 & $0.33(0.64)$ & 2.14 & 4.25 & .20 \\
\hline & $1.99(0.91)$ & -0.60 & -0.44 & .48 & $2.02(0.96)$ & -0.60 & -0.69 & .43 & $2.00(0.81)$ & -0.68 & -0.07 & .49 \\
\hline & $2.40(0.77)$ & -1.03 & 0.16 & .72 & 2.21 & -0.90 & -0.03 & .77 & $2.30(0.73)$ & -0.92 & 0.28 & .78 \\
\hline 35 & $2.43(0.81)$ & -1.32 & 0.97 & .39 & $1.91(1.03)$ & -0.43 & -1.05 & .49 & $2.17(0.78)$ & -0.73 & -0.10 & .45 \\
\hline Ítem 36 & $2.17(0.84)$ & -0.78 & -0.02 & .69 & $1.89(0.88)$ & -0.37 & -0.63 & .66 & $2.02(0.74)$ & -0.48 & -0.25 & .70 \\
\hline Ítem 37 & $2.15(0.92)$ & -0.76 & -0.49 & .41 & $1.62(1.03)$ & -0.07 & -1.16 & .38 & $1.89(0.83)$ & -0.38 & -0.63 & .39 \\
\hline Ítem 38 & $1.80(1.08)$ & -0.34 & -1.19 & .49 & $1.67(1.10)$ & -0.21 & -1.29 & .51 & $1.74(1.02)$ & -0.30 & -1.14 & .52 \\
\hline Ítem 39 & $2.35(0.76)$ & -1.01 & 0.57 & .71 & $2.01(0.87)$ & -0.53 & -0.49 & .76 & $2.17(0.72)$ & -0.68 & 0.06 & .75 \\
\hline & $2.53(0.70)$ & -1.41 & 1.38 & .70 & $2.36(0.83)$ & -1.11 & 0.38 & .74 & $2.44(0.68)$ & -1.09 & 0.50 & .74 \\
\hline Ítem 41 & $2.02(0.89)$ & -0.47 & -0.73 & .60 & $1.68(0.95)$ & -0.04 & -1.00 & .56 & $1.85(0.78)$ & -0.22 & -0.42 & .58 \\
\hline Ítem 42 & $2.34(0.92)$ & -1.28 & 0.58 & .33 & $1.75(1.13)$ & -0.30 & -1.33 & .50 & $2.05(0.84)$ & -0.66 & -0.20 & .43 \\
\hline
\end{tabular}

Nota: $A$ = Asimetria; $C=$ Curtosis; $\mathrm{IH}_{\mathrm{C}}=$ Índice de homogeneidad corregida; La negrita señala los ítems que fueron conservados luego del análisis factorial 
Tabla 2.

Estadísticos descriptivos para las versiones de EPP-ADO.

\begin{tabular}{|c|c|c|c|c|c|c|c|c|c|c|c|c|}
\hline \multirow[b]{2}{*}{ EPP-ADU } & \multicolumn{4}{|c|}{ MADRE } & \multicolumn{4}{|c|}{ PADRE } & \multicolumn{4}{|c|}{ PROMEDIO } \\
\hline & $M(D E)$ & $A$ & C & $\mathrm{IH}_{\mathrm{C}}$ & $M(D E)$ & $A$ & C & $\mathrm{IH}_{\mathrm{C}}$ & E) & $A$ & C & $\mathrm{IH}_{\mathrm{C}}$ \\
\hline 16 & 9) & .35 & 3 & .67 & & 8 & 06 & .70 & 0) & -1.07 & 1 & .71 \\
\hline & & 0.73 & 35 & .48 & 2) & -0.94 & 0.29 & .51 & .63) & -0.93 & 1.05 & .50 \\
\hline & .83) & -1.09 & 0.51 & .65 & $12(0.94)$ & -0.74 & -0.49 & .67 & $.21(0.79)$ & -0.82 & 0.01 & .70 \\
\hline Ítem 4 & $.16(0.87)$ & -0.75 & -0.26 & .65 & $2.85(0.95)$ & -0.38 & -0.81 & .69 & $2.99(0.81)$ & -0.56 & -0.28 & .69 \\
\hline Íten & $1.70(0.92)$ & 1.11 & 0.19 & .44 & $1.72(0.91)$ & 1.09 & 0.19 & .50 & $1.72(0.82)$ & 1.08 & 0.39 & .44 \\
\hline & $3.09(0.78)$ & -0.73 & 0.37 & .53 & 2.98(0.85) & -0.59 & -0.19 & .63 & $3.02(0.73)$ & -0.68 & 0.39 & .60 \\
\hline & 2.31( & 0.22 & -0.98 & .55 & .01) & 0.20 & -1.06 & .59 & $2.32(0.90)$ & 0.14 & -0.88 & .56 \\
\hline Íter & 3.44 & -1.36 & 1.33 & .20 & 86) & -1.24 & 0.69 & .28 & $3.40(0.74)$ & -1.24 & 1.11 & .24 \\
\hline Íter & 3.50( & -1.37 & 1.48 & .60 & 3.33 & -1.13 & 0.72 & .64 & $3.40(0.67)$ & -1.10 & 0.89 & .65 \\
\hline Íter & 2.53( & 0.06 & -1.01 & .48 & $2.40(1.05)$ & 0.17 & -1.18 & .44 & $2.47(0.87)$ & 0.10 & -0.80 & .44 \\
\hline İter & $3.27(0.83)$ & -0.93 & 0.07 & .33 & $3.03(0.96)$ & -0.67 & -0.56 & .36 & $3.15(0.79)$ & -0.78 & 0.00 & .35 \\
\hline Íten & $3.13(0.89)$ & -0.64 & -0.60 & .62 & $2.63(0.99)$ & -0.06 & -1.06 & .60 & $2.88(0.82)$ & -0.32 & -0.67 & .64 \\
\hline İter & 3.23 & -0.77 & 0.05 & .46 & $3.09(0.86)$ & -0.56 & -0.56 & .46 & $3.15(0.71)$ & -0.55 & -0.15 & .42 \\
\hline İter & $3.28(0.87)$ & -0.98 & 0.04 & .74 & $2.88(0.95)$ & -0.40 & -0.82 & .69 & $3.08(0.80)$ & -0.68 & -0.13 & .72 \\
\hline Íter & $2.85(1.02)$ & -0.44 & -0.94 & .44 & $2.43(1.07)$ & 0.18 & -1.22 & .46 & $2.64(0.95)$ & -0.16 & -0.95 & .45 \\
\hline & $3.61(0.72)$ & -1.97 & 3.40 & .54 & $3.48(0.86)$ & -1.67 & 1.89 & .55 & $3.53(0.72)$ & -1.72 & 2.58 & .55 \\
\hline & $1.64(0.92)$ & 1.29 & 0.52 & .43 & $1.66(0.96)$ & 1.29 & 0.46 & .47 & $1.66(0.84)$ & 1.15 & 0.37 & .44 \\
\hline & $3.25(0.77)$ & -0.74 & -0.13 & .72 & $2.81(0.89)$ & -0.24 & -0.78 & .70 & $3.03(0.72)$ & -0.43 & -0.24 & .72 \\
\hline Íten & $2.83(0.93)$ & -0.32 & -0.81 & .52 & $2.43(0.99)$ & 0.13 & -1.02 & .55 & $2.64(0.84)$ & -0.20 & -0.60 & .53 \\
\hline & $2.60(0.92)$ & 0.01 & -0.88 & .59 & $2.58(1.00)$ & -0.00 & -1.08 & .63 & $2.59(0.84)$ & -0.15 & -0.70 & .61 \\
\hline & $3.12(0.93)$ & -0.68 & -0.63 & .71 & $2.70(0.99)$ & -0.06 & -1.14 & .68 & $2.91(0.87)$ & -0.37 & -0.74 & .71 \\
\hline & $2.53(0.93)$ & 0.02 & -0.88 & .61 & 2.45(0.99) & 0.10 & -1.02 & .65 & $2.49(0.87)$ & -0.01 & -0.72 & .64 \\
\hline 23 & 3.331 & -1.13 & 0.53 & .67 & $3.19(0.91)$ & -0.92 & -0.06 & .67 & $3.25(0.80)$ & -1.03 & 0.50 & .69 \\
\hline & 1.54( & 1.53 & 1.28 & .29 & $1.53(0.85)$ & 1.50 & 1.22 & .35 & $1.54(0.78)$ & 1.39 & 1.15 & .30 \\
\hline & $2.84(1.04)$ & -0.44 & -1.00 & .29 & $2.03(0.99)$ & 0.56 & -0.80 & .33 & $2.45(0.88)$ & -0.02 & -0.76 & .34 \\
\hline & 3.21 & -0.89 & -0.04 & .77 & 2.73 & -0.19 & -1.06 & .75 & 2.9810 & -0.52 & -0.45 & .78 \\
\hline & 6) & -1.01 & 0.11 & .81 & 2.92 & -0.48 & -0.83 & .77 & 3.1 & -0.70 & -0.20 & .82 \\
\hline & 2.5 & -0.07 & -0.98 & .60 & 2.44 & 0.03 & -1.23 & .62 & 2.4 & -0.06 & -0.97 & .62 \\
\hline & 1) & -0.66 & -0.60 & .73 & 2.71 & -0.14 & -1.07 & .67 & 2.9 & -0.40 & -0.68 & .72 \\
\hline & i & -1.08 & 0.44 & .07 & 3.25 & -1.01 & 0.13 & .19 & $3.2 \varepsilon$ & -0.94 & 0.25 & .12 \\
\hline & 3.35 & -1.08 & 0.56 & .69 & 3.03 & -0.67 & -0.30 & .72 & $3.19(0.76)$ & -0.78 & 0.18 & .73 \\
\hline & $1.77(0.96)$ & 1.01 & -0.09 & .45 & 1.73( & 1.12 & 0.15 & .52 & $1.75(0.86)$ & 0.99 & 0.06 & .48 \\
\hline 33 & $2.95(0.91)$ & -0.47 & -0.65 & .46 & $2.95(0.96)$ & -0.51 & -0.68 & .40 & $2.94(0.82)$ & -0.51 & -0.33 & .46 \\
\hline 34 & $3.33(0.83)$ & -1.09 & 0.42 & .76 & $3.13(0.93)$ & -0.80 & -0.34 & .77 & $3.23(0.79)$ & -0.85 & 0.04 & .80 \\
\hline & $3.26(0.84)$ & -0.97 & 0.23 & .40 & $2.71(1.02)$ & -0.16 & -1.15 & .51 & $3.00(0.80)$ & -0.46 & -0.37 & .50 \\
\hline 36 & $3.24(0.81)$ & -0.87 & 0.13 & .73 & $2.94(0.89)$ & -0.49 & -0.55 & .71 & $3.08(0.75)$ & -0.52 & -0.29 & .76 \\
\hline 37 & $3.02(0.90)$ & -0.51 & -0.69 & .37 & $2.48(1.05)$ & 0.11 & -1.19 & .44 & $2.77(0.85)$ & -0.14 & -0.80 & .41 \\
\hline 38 & 2.93(0.97) & -0.50 & -0.79 & .34 & $2.81(1.02)$ & -0.37 & -1.00 & .42 & $2.86(0.94)$ & -0.42 & -0.80 & .38 \\
\hline Ítem 39 & $3.35(0.77)$ & -1.01 & 0.42 & .74 & $3.01(0.87)$ & -0.43 & -0.71 & .74 & $3.18(0.73)$ & -0.58 & -0.34 & .78 \\
\hline & $3.43(0.79)$ & -1.20 & 0.57 & .71 & $3.27(0.89)$ & -1.06 & 0.22 & .71 & $3.34(0.77)$ & -1.05 & 0.40 & .74 \\
\hline Íte & $2.87(0.95)$ & -0.33 & -0.91 & .66 & $2.54(0.96)$ & 0.08 & -0.97 & .68 & $2.70(0.86)$ & -0.11 & -0.72 & .70 \\
\hline Ítem 42 & $3.19(0.91)$ & -0.85 & -0.23 & .43 & $2.43(1.09)$ & 0.16 & -1.28 & .49 & $2.82(0.84)$ & -0.25 & -0.60 & .47 \\
\hline
\end{tabular}

Nota: $A$ = Asimetría; $C=$ Curtosis; $\mathrm{IH}_{\mathrm{C}}=$ Índice de homogeneidad corregida; La negrita señala los ítems que fueron conservados luego del análisis factorial

\section{Análisis de datos}

Primero se procedió a estudiar la distribución de los ítems y su índice de homogeneidad total-corregida. En la tabla 1 pueden visualizarse los datos relacionados a la versión EPP-ADO y en la tabla 2 aquellos de la versión EPPADU. Los valores se encontraron dentro de los parámetros esperados. En cuanto a los datos perdidos, estos no superaban el umbral del $30 \%$, por lo que su tratamiento fue con el procedimiento de estimación por lista. 
Para estudiar la dimensionalidad de la medida se utilizó el software FACTOR en su versión 10.03.01 (Lorenzo-Seva \& Ferrando, 2013). Dado que los ítems se respondieron en una escala likert, se utlizó la matriz policórica y el análisis paralelo se basó en el análisis factorial de rango mínimo (MRFA; Timmerman \& Lorenzo-Seva, 2011). Como se esperaba que los factores fueran dimensiones separadas poco relacionadas, se utilizó la rotación Varimax. Para obtener una estructura que fuera adecuada y consistente con la teoría y versátil para la valoración de las dinámicas familiares actuales, se estableció que la estructura debería contar con las siguientes caracteristicas: (1) ítems con cargas factoriales altas (>.40) en un solo factor; (2) los factores debian tener al menos cinco items; (3) la consistencia interna de los factores debía ser alta (>.80); y (4) la estructura debia ser idéntica en todas las versiones del test (ambos progenitores, el promedio de respuestas y en ambas muestras). Luego, se procedió a realizar estudios confirmatorios de validación cruzada e invarianza factorial para dar mayor sustento al uso de la medida en sus distintas versiones. Para el análisis de correlaciones con los distintos criterios externos, se calcularon correlaciones $r$ de Pearson.

\section{RESULTADOS}

\section{Análisis Factorial Exploratorio y consistencia interna}

Se realizaron análisis factoriales exploratorios por separado para la muestra de adolescentes y para la muestra de adultos. Además, se analizaron por separado las distintas versiones del test. Se denominó MADRE a las respuestas relacionadas con el adulto mayormente a cargo, dado que en la mayoria de los casos los evaluados informaron estar refiriéndose a su madre (95.9\% en el caso de los adolescentes y 99.4\% en el caso de los adultos), se denominó PADRE a las respuestas relacionadas con el segundo adulto a cargo, dado que en la mayoría de los casos los evaluados informaron estar refiriéndose a su padre $(98.9 \%$ en el caso de los adolescentes y $99.2 \%$ en el caso de los adultos) y se denominó PROMEDIO a las respuestas promediadas entre las versiones MADRE y PADRE para validar una estructura factorial viable en el caso de que el interés sea una única medida conjunta de la percepción de parentalidad por parte del evaluado.

Al calcularse los análisis factoriales exploratorios, en todos los casos el análisis paralelo sugirió la extracción de dos factores, lo cual era coincidente con la teoría sobre la parentalidad. Se analizaron en simultáneo todas las versiones del test, y se buscó una estructura factorial en común, lo que llevó a la eliminación de 22 ítems. La EPP quedó conformada así por 20 ítems repartidos en dos dimensiones correspondientes con la teoria: respuesta y demanda.

En el caso de la versión para adolescentes (EPP-ADO), la estructura aislada para la versión MADRE (o adulto mayormente a cargo) explicaba el $54.33 \%$ de la varianza $(K M O=.89$, Bartlett $=2492.3, p<.001)$, la versión PADRE (o segundo adulto a cargo) explicaba $58.08 \%$ de la varianza $(K M O=$ .90 , Bartlett $=4098.0, p<.001$ ), y la versión PROMEDIO (combinación de puntajes en el caso de que haya informado sobre dos progenitores y se quiera analizar una medición conjunta) explicaba el $56.66 \%$ de la varianza $(K M O=$ .90$, Bartlett $=3972.1, p<.001)$. En el caso de la versión para adultos (EPPADU), la estructura aislada para la versión MADRE (o adulto mayormente a 
cargo) explicaba el 56.24\% de la varianza $(K M O=.89$. Bartlett $=4568.9, p<$ .001 ), la versión PADRE (o segundo adulto a cargo) explicaba el 56.13\% de la varianza $(K M O=.89$, Bartlett $=4360.2, p<.001)$, y la versión PROMEDIO (combinación de puntajes en el caso de que haya informado sobre dos progenitores y se quiera analizar una medición conjunta) explicaba el $68.91 \%$ de la varianza $(K M O=.89$, Bartlett $=4563.4, p<.001)$. En las tablas 3 y 4 se pueden visualizar las cargas factoriales de cada versión del test en la muestra de adolescentes y en la muestra de adultos, la varianza explicada por cada factor y las medidas de consistencia interna (alfas de Cronbach y Omega). Todos los items presentaron cargas superiores a .40 en un único factor, a excepción del ítem "Me exigía que cuidara las cosas de la casa" para la versión PROMEDIO para adultos que presentó una carga de .39. Se decidió conservarlo por ser un valor limítrofe y dado que sus cargas en las otras versiones eran las esperadas. Los valores de consistencia interna tanto de los alfas de Crobach como de los Omegas indicaron una excelente consistencia interna para todas las escalas (ver tablas 3 y 4).

Tabla 3.

Análisis factoriales exploratorios de la EPP en muestra de adolescentes.

\begin{tabular}{|c|c|c|c|c|c|c|}
\hline \multirow[b]{3}{*}{ ITEM } & \multicolumn{6}{|c|}{ Versión EPP-ADO } \\
\hline & \multicolumn{2}{|c|}{ MADRE } & \multicolumn{2}{|c|}{ PADRE } & \multicolumn{2}{|c|}{ PROMEDIO } \\
\hline & 1 & 2 & 1 & 2 & 1 & 2 \\
\hline Me abraza & .08 & .79 & .79 & .12 & .13 & .79 \\
\hline Me protege cuando lo necesito & .12 & .85 & .86 & .09 & .13 & .86 \\
\hline Me exige que le cuente con quienes salgo & .52 & .11 & .23 & .56 & .56 & .15 \\
\hline Me felicita cuando hago las cosas bien & .11 & .73 & .76 & .04 & .06 & .75 \\
\hline Controla mis horarios & .61 & .13 & .20 & .70 & .66 & .19 \\
\hline Me castiga si no le obedezco & .55 & .01 & -.09 & .56 & .55 & -.01 \\
\hline Pone límites estrictos & .76 & -.08 & -.02 & .84 & .78 & -.04 \\
\hline Está disponible para hablar conmigo & -.01 & .81 & .80 & -.06 & -.05 & .80 \\
\hline Me ayuda cuando lo necesito & -.06 & .81 & .77 & .08 & .03 & .81 \\
\hline Me prohíbe cosas si me porto mal & .68 & -.05 & -.06 & .81 & .73 & -.07 \\
\hline Está atento a lo que me ocurre & .15 & .81 & .82 & .17 & .19 & .82 \\
\hline Me pide que ayude con tareas del hogar & .55 & .15 & .24 & .46 & .45 & .22 \\
\hline Me dice que me quiere & .10 & .76 & .79 & .13 & .18 & .77 \\
\hline Me exige que cuide las cosas de la casa & .52 & .34 & .29 & .53 & .48 & .36 \\
\hline Pretende que ordene mi habitación & .47 & .18 & .18 & .60 & .54 & .21 \\
\hline Me reta si me porto mal & .66 & .17 & .10 & .68 & .66 & .22 \\
\hline Me contiene cuando estoy triste & .05 & .83 & .84 & .02 & .05 & .84 \\
\hline Me escucha & -.05 & .88 & .86 & -.02 & -.02 & .86 \\
\hline Pretende que se cumplan sus reglas & .60 & .05 & .08 & .64 & .60 & .18 \\
\hline Me alienta & .09 & .81 & .85 & .07 & .10 & .84 \\
\hline \% Varianza explicada & 23.6 & 43.6 & 44.5 & 27.4 & 25.9 & 47.2 \\
\hline Alfa de Cronbach & .79 & .91 & .93 & .83 & .81 & .93 \\
\hline Omega & .85 & .95 & .95 & .86 & .85 & .95 \\
\hline
\end{tabular}


Tabla 4.

Análisis factoriales exploratorios de la EPP en muestra de adultos.

\begin{tabular}{|c|c|c|c|c|c|c|}
\hline \multirow[b]{3}{*}{ ITEM } & \multicolumn{6}{|c|}{ Versión EPP-ADU } \\
\hline & \multicolumn{2}{|c|}{ MADRE } & \multicolumn{2}{|c|}{ PADRE } & \multicolumn{2}{|c|}{ PROMEDIO } \\
\hline & 1 & 2 & 1 & 2 & 1 & 2 \\
\hline Me abrazaba & .81 & .05 & .77 & -.01 & .77 & .03 \\
\hline Me protegía cuando lo necesitaba & .82 & .11 & .81 & .05 & .82 & .06 \\
\hline Me exigía que le cuente con quienes salía & .18 & .55 & .20 & .52 & .15 & .56 \\
\hline Me felicitaba cuando hacía las cosas bien & .79 & .01 & .78 & -.01 & .76 & -.01 \\
\hline Controlaba mis horarios & .13 & .72 & .20 & .69 & .15 & .72 \\
\hline Me castigaba si no le obedecía & -.13 & .59 & -.24 & .66 & -.15 & .60 \\
\hline Ponía límites estrictos & -.09 & .76 & -.01 & .75 & -.02 & .72 \\
\hline Estaba disponible para hablar conmigo & .84 & -.05 & .78 & .03 & .78 & .01 \\
\hline Me ayudaba cuando lo necesitaba & .71 & .04 & .72 & .03 & 69 & .03 \\
\hline Me prohibía cosas si me portaba mal & -.06 & .68 & -.07 & .76 & -.06 & .73 \\
\hline Estaba atento a lo que me ocurría & .84 & .11 & .83 & .12 & .83 & .11 \\
\hline Me pedía que ayude con tareas del hogar & .02 & .51 & .10 & .56 & .08 & .45 \\
\hline Me decía que me quería & .79 & .02 & .77 & -.01 & .78 & .05 \\
\hline Me exigía que cuidara las cosas de la casa & .21 & .47 & .16 & .47 & .23 & .39 \\
\hline Pretendía que ordene mi habitación & .17 & .61 & .22 & .60 & .22 & .55 \\
\hline Me retaba si me portaba mal & .19 & .56 & -.01 & .59 & .14 & .47 \\
\hline Me contenía cuando estaba triste & .86 & .05 & .86 & .07 & .85 & .11 \\
\hline Me escuchaba & .92 & -.06 & .86 & -.03 & .89 & -.05 \\
\hline Pretendía que se cumplan sus reglas & -.02 & .65 & -.01 & .71 & .07 & .59 \\
\hline Me alentaba & .87 & .03 & .85 & .01 & .87 & .04 \\
\hline$\%$ Varianza explicada & 45.2 & 24.5 & 43.6 & 26.6 & 45.2 & 23.7 \\
\hline Alfa de Cronbach & .93 & .80 & .92 & .82 & .93 & .81 \\
\hline Omega & .95 & .85 & .95 & .87 & .95 & .84 \\
\hline
\end{tabular}

\section{Análisis Factorial Confirmatorio e invarianza factorial}

Luego se realizaron análisis factoriales confirmatorios para todas las escalas. El método de estimación fue Máxima Verosimilitud Robusto con base en la matriz policórica de los datos. Como puede visualizarse en la tabla 5, todas las estructuras demostraron poseer un adecuado ajuste. Las cargas factoriales fueron superiores a .40 a excepción del item "Me exigia que cuidara las cosas de la casa" que obtuvo una carga de valor limítrofe (.38) en la versión PROMEDIO para adultos.

Tabla 5.

Indicadores de ajuste de los análisis factoriales confirmatorio de la EPP.

\begin{tabular}{lcccccc}
\hline & S-B $\boldsymbol{X}^{\mathbf{2}}(\mathbf{g l})$ & CFI & NFI & IFI & RMSEA & IC 90\% RMSEA \\
\cline { 2 - 7 } EPP-ADO & & & & & & \\
MADRE & $469.04(169)^{* *}$ & .960 & .939 & .960 & .064 & $(.057-.070)$ \\
PADRE & $564.15(169)^{* *}$ & .959 & .943 & .959 & .074 & $(.067-.080)$ \\
PROMEDIO & $535.53(169)^{* *}$ & .963 & .946 & .963 & .071 & $(.065-.078)$ \\
EPP-ADU & & & & & & \\
MADRE & $588.84(169)^{* *}$ & .956 & .940 & .957 & .070 & $(.064-.076)$ \\
PADRE & $678.84(169)^{* *}$ & .946 & .929 & .946 & .079 & $(.073-.086)$ \\
PROMEDIO & $603.03(169)^{* *}$ & .950 & .932 & .950 & .074 & $(.067-.080)$ \\
\hline
\end{tabular}

Nota: ${ }^{* *} p<.001$ 
A continuación, se calculó una prueba de invarianza factorial entre las versiones EPP-ADO MADRE, EPP-ADO PADRE, EPP-ADU MADRE y EPP-ADU PADRE. La versión PROMEDIO no fue incluida en este análisis para no incumplir con el supuesto estadístico de independencia de los datos analizados. Primero se estimó un modelo en el que no se establecieron restricciones de invarianza (Modelo 1), luego uno en donde se estableció la invarianza de las regresiones (Modelo 2) y, finalmente, uno en donde se sumó la restricción de invarianza en las covarianzas (Modelo 3). En todos los casos el ajuste global valorado por el CFI fue adecuado y de nula variación entre modelos. Además, la prueba de invarianza entre los valores de Satorra-Bentler no fue estadísticamente significativa en ninguno de los contrastes entre modelos $(\Delta \mathrm{S}-\mathrm{B} p>$.005), por lo que puede interpretarse la invarianza de la estructura factorial entre las cuatro versiones del test. En la tabla 6 pueden visualizarse los datos sobre los tres modelos de invarianza factorial.

Tabla 6.

Invarianza factorial entre las versiones MADRE y PADRE de adolescentes y adultos.

\begin{tabular}{|c|c|c|c|c|c|c|c|c|}
\hline & S-B(gl) & CFI & RMSEA (IC 90\%) & $\begin{array}{l}\text { Comparación } \\
\text { de modelos }\end{array}$ & $\Delta \mathrm{S}-\mathrm{B}$ & $\Delta \mathrm{gl}$ & $p$ & $\Delta \mathrm{CFI}$ \\
\hline $\begin{array}{l}\text { Modelo } 1 \\
\text { Sin restricciones } \\
\text { Modelo } 2\end{array}$ & $2288.59(676)$ & .955 & $.072(.069-.075)$ & & & & & \\
\hline $\begin{array}{l}\text { Restricción de cargas } \\
\text { factoriales }\end{array}$ & $2354.02(728)$ & .955 & $.070(.066-.073)$ & 2 vs. 1 & 62.89 & 52 & .143 & 0 \\
\hline $\begin{array}{l}\text { Modelo } 3 \\
\text { Restricción de cargas } \\
\text { factoriales y covarianzas }\end{array}$ & $2357.70(731)$ & .955 & $.069(.066-.072)$ & 3 vs. 2 & 3.23 & 3 & .356 & 0 \\
\hline
\end{tabular}

\section{Estilos Parentales}

Para el cálculo de los estilos parentales se utilizó la clasificación de Maccoby y Martin (1983). Para ello se empleó el puntaje de corte T en el valor de 50 en las dimensiones de respuesta y demanda y se calcularon los cuatro estilos que propusieron los autores. La combinación y resultados obtenidos en términos de frecuencia se pueden observar en la tabla 7. El estilo más frecuente en todos los casos fue el autoritativo y el menos frecuente fue el autoritario.

Tabla 7.

Porcentajes de estilos parentales en las distintas versiones de la EPP.

\begin{tabular}{lcccc}
\hline & Negligente & Autoritario & Permisivo & Autoritativo \\
\cline { 2 - 5 } Adolescentes & & & & \\
Madre & 24.6 & 15.3 & 27.6 & 32.6 \\
Padre & 24.7 & 19.1 & 24.0 & 32.3 \\
Promedio & 26.0 & 19.4 & 23.0 & 31.6 \\
Adultos & & & & \\
Madre & 22.3 & 19.5 & 29.0 & 29.2 \\
Padre & 24.6 & 20.5 & 24.0 & 31.0 \\
Promedio & 22.9 & 22.5 & 23.2 & 31.4 \\
\hline
\end{tabular}

\section{Asociaciones con criterios externos}

A continuación, se procedió a estudiar el comportamiento de las dimensiones en relación con tres criterios externos: afrontamiento del estrés, 
sintomatología psicológica y bienestar. En ambas muestras se midió la frecuencia de uso de estilos de afrontamiento, en la muestra de adolescentes se contaba con una medida de sintomatología psicológica y en la muestra de adultos se contaba con una medida de bienestar. En las tablas 8 y 9 pueden apreciarse las correlaciones obtenidas mediante pruebas $r$ de Pearson.

En el caso de los adolescentes, la aproximación cognitiva se asoció positiva y débilmente con la respuesta parental promedio. El afrontamiento por aproximación conductual estaba asociado positiva pero débilmente a la respuesta parental materna y promedio, y la evitación cognitiva se asociaba de manera inversa y débil también. En todos los casos, la asociación entre la respuesta parental y la sintomatología era negativa entre débil y moderada. La única asociación significativa para la dimensión demanda fue positiva y débil en relación con la evitación cognitiva y solo en el caso de la demanda materna.

Tabla 8.

Muestra de adolescentes: asociaciones entre las dimensiones de parentalidad, afrontamiento y sintomatología.

\begin{tabular}{lcccccc}
\hline & $\begin{array}{c}\text { Respuesta } \\
\text { materna }\end{array}$ & $\begin{array}{c}\text { Demanda } \\
\text { materna }\end{array}$ & $\begin{array}{c}\text { Respuesta } \\
\text { paterna }\end{array}$ & $\begin{array}{c}\text { Demanda } \\
\text { paterna }\end{array}$ & $\begin{array}{c}\text { Respuesta } \\
\text { promedio }\end{array}$ & $\begin{array}{c}\text { Demanda } \\
\text { promedio }\end{array}$ \\
\cline { 2 - 7 } Aproximación Cognitiva & .08 & .07 & .07 & .03 & $.09^{*}$ & .06 \\
Aproximación Conductual & $.09^{*}$ & -.00 & .06 & -.01 & $.10^{*}$ & -.01 \\
Evitación Cognitiva & -.06 & $.09^{*}$ & $-.13^{* *}$ & .02 & $-.11^{*}$ & .07 \\
Evitación Conductual & .01 & .01 & .06 & -.03 & .05 & -.00 \\
Sintomatología Psicológica & $-.18^{* *}$ & -.02 & $-.30^{* *}$ & -.06 & $-.27^{* *}$ & -.04 \\
\hline
\end{tabular}

Nota: ${ }^{*} p<.05,{ }^{* *} p<.01$

En el caso de los adultos, la respuesta materna y la respuesta promedio se asociaron negativa y débilmente con la evitación cognitiva. Además, todas las mediciones de respuesta parental se asociaron positivamente con la presencia de bienestar total y todas sus subescalas. Además, la demanda materna y promedio se asoció positivamente con la presencia de bienestar social.

Tabla 9.

Muestra de adultos: asociaciones entre las dimensiones de parentalidad, afrontamiento y bienestar.

\begin{tabular}{lcccccc}
\hline & $\begin{array}{c}\text { Respuesta } \\
\text { materna }\end{array}$ & $\begin{array}{c}\text { Demanda } \\
\text { materna }\end{array}$ & $\begin{array}{c}\text { Respuesta } \\
\text { paterna }\end{array}$ & $\begin{array}{c}\text { Demanda } \\
\text { paterna }\end{array}$ & $\begin{array}{c}\text { Respuesta } \\
\text { promedio }\end{array}$ & $\begin{array}{c}\text { Demanda } \\
\text { promedio }\end{array}$ \\
\cline { 2 - 7 } Aproximación Cognitiva & -.03 & .04 & .02 & .03 & .00 & .04 \\
Aproximación Conductual & -.03 & .06 & .03 & .03 & .01 & .06 \\
Evitación Cognitiva & $-.11^{* *}$ & .04 & -.08 & .01 & $-.12^{* *}$ & .02 \\
Evitación Conductual & .02 & .05 & .04 & .05 & .04 & .05 \\
Bienestar total & $.10^{*}$ & .04 & $.13^{* *}$ & .07 & $.14^{* *}$ & .06 \\
Bienestar emocional & $.18^{* *}$ & -.05 & $.14^{* *}$ & .04 & $.18^{* *}$ & -.01 \\
Bienestar psicológico & $.10^{*}$ & .02 & $.10^{*}$ & .03 & $.12^{* *}$ & .03 \\
Bienestar social & .01 & $.09^{*}$ & .08 & .08 & .06 & $.10^{*}$ \\
\hline
\end{tabular}

Nota: ${ }^{*} p<.05,{ }^{* \star} p<.01$

Luego, se calcularon ANOVAs one-way con pruebas pos-hoc Bonferroni para analizar si existian diferencias en los criterios externos de acuerdo a los estilos parentales. En adolescentes se encontró mayor presencia de evitación cognitiva en los adolescentes que tenían madres, $F(3,435)=3.73, p=.011$, y padres $F(3,426)=4.36, p=.005$-así como también en la diada (promedio) $F(3,439)=4.56, p=.004-$ con estilos autoritarios en comparación con estilos 
permisivos; y mayor presencia de evitación conductual en los adolescentes que tenian padres con estilos permisivos en comparación con los estilos autoritativos. A su vez, más presencia de aproximación conductual en los que la diada parental (promedio) tenían un estilo permisivo en comparación con un estilo negligente $F(3,439)=2.63, p=.049$. Además, se evidenció mayor sintomatología en quienes tenían madres, $F(3,435)=5.21, p=.002$, y padres, $F(3,426)=9.99, p<.001$, -así como también en la díada (promedio), $F(3,439)$ $=8.27, p<.001-$ con estilos autoritarios y negligentes en comparación con los estilos permisivos y autoritativos. En el caso de los adultos solo de verificó una diferencia estadisticamente significativa en la presencia de bienestar emocional $F(3,503)=4.76, p=.003$. Quienes tenian madres y padres con estilos autoritarios tenían menos bienestar emocional que los que tenían madres con estilos permisivos y autoritativos.

\section{Baremización}

Con el objetivo de brindar herramientas para el uso de la técnica en el ámbito de aplicación, se procedió a calcular los baremos correspondientes a cada escala utilizando puntuaciones T. En ella, se brindan los puntajes de corte para valorar las dimensiones individualmente, como así también para su combinación en estilos parentales de acuerdo con la clasificación de Maccoby y Martin (1983).

En el caso de la evaluación dimensional de cada escala, se debe seguir la siguiente pauta: (a) puntajes $\geq \mathrm{T} 55$ se consideran un alta presencia de la dimensión evaluada; (b) puntajes entre el valor inmediatamente inferior a del T55 e inmediatamente superior al del T45 se consideran una presencia término medio; y (c) puntajes $\leq \mathrm{T} 45$ indican una presencia inferior a la esperada en la dimensión evaluada.

Para el cálculo de los estilos parentales se debe seguir la siguiente combinación de puntajes: (a) puntajes < T50 en respuesta y puntajes < T50 configuran un estilo negligente; (b) puntajes < T50 en respuesta y $\geq \mathrm{T} 50$ en demanda un estilo autoritario; (c) puntajes $\geq$ T50 en respuesta y puntajes $<$ T50 en demanda un estilo permisivo; y (d) puntajes $\geq$ T50 en respuesta y puntajes $\geq$ T50 en demanda un estilo autoritativo. En la tabla 10 se informan los baremos obtenidos.

Tabla 10.

Baremos de la EPP: puntajes de corte para las versiones adolescentes y adultos.

\begin{tabular}{|c|c|c|c|c|c|c|c|c|c|c|c|c|c|}
\hline \multirow[b]{3}{*}{$\mathbf{T}$} & \multicolumn{6}{|c|}{ EPP-ADO } & \multicolumn{7}{|c|}{ EPP-ADU } \\
\hline & \multicolumn{2}{|c|}{ MADRE } & \multicolumn{2}{|c|}{ PADRE } & \multicolumn{2}{|c|}{ PROMEDIO } & \multicolumn{2}{|c|}{ MADRE } & \multicolumn{2}{|c|}{ PADRE } & \multicolumn{2}{|c|}{ PROMEDIO } & \\
\hline & $\mathbf{R}$ & D & $\mathbf{R}$ & D & $\mathbf{R}$ & D & $\mathbf{R}$ & D & $\mathbf{R}$ & D & $\mathbf{R}$ & D & $T$ \\
\hline 55 & 2.66 & 2.17 & 2.41 & 1.93 & 2.50 & 2.02 & 2.6 & 2.16 & 2.32 & 1.93 & 2.43 & 2.02 & 55 \\
\hline 50 & 2.38 & 1.92 & 2.08 & 1.63 & 2.23 & 1.78 & 2.29 & 1.91 & 1.98 & 1.65 & 2.14 & 1.78 & 50 \\
\hline 45 & 2.11 & 1.67 & 1.76 & 1.34 & 1.96 & 1.55 & 2.00 & 1.66 & 1.66 & 1.37 & 1.86 & 1.56 & 45 \\
\hline
\end{tabular}

\section{DISCUSIÓN}

El objetivo general de esta investigación radicaba en generar una medida psicométrica para medir parentalidad percibida en adolescentes y adultos que fuera versátil en términos de comparabilidad entre esa población y de 
contemplación de configuraciones familiares tradicionales y nuevas. Las estructuras factoriales aisladas en los análisis exploratorios replican la bidimensionalidad esperada de respuesta y demanda (Baumrind, 1996). En un factor se agruparon items que valoran la presencia de afecto, contención, aliento y escucha -dimensión respuesta- y en el otro, items relacionados con la puesta de limites, exigencias y control -dimensión demanda-. Todas las estructuras tenian una apropiada adecuación muestral, explicaban un porcentaje de varianza apropiado y las dimensiones aisladas tenian una buena consistencia interna. Luego, los análisis confirmatorios y de invarianza dieron mayor sustento a la estructura y además son habilitantes para realizar comparaciones de resultados utilizando las distintas versiones del test.

En cuanto al estudio del comportamiento de las variables medidas con la EPP y dos criterios externos clásicamente analizados en el estudio de la parentalidad, puede decirse que los resultados dan aún mayor sustento a la calidad de la medida, ya que replican estudios previos tanto en cuanto a las relaciones con sintomatología psicológica (Chyung \& Lee, 2008; Dwairy \& Dor, 2009; Khaleque \& Rohner, 2012; Ripoll-Nuñez \& Álvarez, 2008), como a la presencia de bienestar (Aguilar Villalobos et al. 2003; Schwarz et al., 2011) y al uso de estrategias de afrontamiento (de la Iglesia, 2013; Gaylord-Harden et al., 2010; Zhou et al., 2008). Se evidenció que la dimensión de respuesta se encuenta asociada a menor sintomatología psicológica y mayor bienestar, así como a un menor uso de afrontamientos del estrés improductivos. Es decir, tal como se ha reportado en numerosas investigaciones, la dimensión de respuesta parental resulta ser aquella con mayor peso y su presencia está asociada a resultados beneficiosos en la progenie. En este caso en particular, se evidenció que los comportamientos afectuosos, de diálogo, de escucha e interés por los hijos se reflejan en una mayor salud mental tanto en el incremento del bienestar como en la disminución de la sintomatología, y, a su vez, en el menor uso de afrontamientos del estrés que no resultarian efectivos de ser utilizados.

Esta investigación cuenta con algunas limitaciones que deben ser mencionadas. En principio, los criterios externos aquí utilizados contemplaron variables psicológicas no ligadas a la crianza, por lo que el comportamiento de las medidas del test no fue contrastado con otra medida de parentalidad ya ajustada psicométricamente. Además, no se contó con una medida de deseabilidad social para valorar la posible distorsión de respuestas de los evaluados. Finalmente, solo se midieron prácticas parentales sanas, por lo que permanece pendiente el diseño de una medida de parentalidad percibida centrada exclusivamente en prácticas parentales patológicas.

Como con toda nueva medida psicométrica, futuras líneas de investigación deberán brindar mayor sustento a su funcionamiento mediante el análisis de su relación con otros criterios externos relevantes, así como con otras poblaciones y con otros diseños. Sería de especial interés realizar un estudio longitudinal para analizar el comportamiento de la medida a lo largo del tiempo y verificar la estabilidad de la percepción de parentalidad en los evaluados. Las medidas aquí validadas tienen como fortaleza la versatilidad que presentan para su uso en distintas configuraciones parentales dado que: (1) se pueden contestar tanto en relación con un adulto a cargo de la crianza como con dos adultos a cargo de la crianza, analizando esos puntajes por separado o bien en promedio, (2) además, aunque en la muestra analizada la mayoría de los evaluados refirió contestar con relación a su madre cuando se 
le preguntó por el adulto mayormente a cargo y con relación a su padre cuando se solicitó se consigne en relación con un segundo adulto a cargo, la amplitud de la consigna con la que el test fue valorado permite valorar formas familiares en las que la configuración no necesariamente fuera madre-padre, sino también en el caso de familias monoparentales, homoparentales $o$ familias en las que otro adulto sin relación biológica cumpla la función de agente socializador (ej. familias ensambladas). El cálculo de los baremos permitirá la transferencia del instrumento al ámbito de aplicación tanto para el análisis de las dimensiones por separado como por conjunto.

\section{REFERENCIAS}

Aguilar Villalobos, J., Martínez Jiménez, M., \& Valencia Cruz, A. (2003). Patrones parentales e indicadores de comportamiento social en estudiantes universitarios. Red de Investigación Educativa en Sonora, Anuario de Investigaciones Educativas, 7, 251-260.

Ainsworth, M. D. S., Bell, S. M., \& Stayton, D. J. (1971). Individual differences in strange situation behavior of one-year-olds. En H. R. Schaffer (Ed.), The origins of human social relations (pp. 17-58). London: Academic Press.

Anisman, H., Zaharia, M. D., Meaney, M. J., \& Merali, Z. (1998). Do early-life events permanently alter behavioral and hormonal responses to stressors? International Journal of Developmental Neuroscience 16(3-4), 149-164. https://doi.org/10.1016/S0736-5748(98)00025-2

Aquilino, W. S. \& Supple, A. J. (2001). Long-Term Effects of Parenting Practices During Adolescence on Well-Being Outcomes in Young Adulthood. Journal of Family Issues, 22(3), 289-308. https://doi.org/10.1177/019251301022003002

Ausubel, D. P., Balthazar, E. E., Rosenthal, I., Blackman, L. S., Schpoont, S. H., \& Welkowitz, J. (1954). Perceived parent attitudes as determinants of children's ego structure. Child Development, 25(3), 173183. https://doi.org/10.2307/1126334

Baumrind, D. (1966). Effects of authoritative parental control on child behavior. Child Development, 37, 887-907. https://doi.org/10.2307/1126611

Baumrind, D. (1975). The contributions of the family to the development of competence in children. Schizophrenia Bulletin, 14, 12-37. https://doi.org/10.1093/schbul/1.14.12

Baumrind, D. (1991a). The influence of parenting style on adolescent competence and substance use. The Journal of Early Adolescence, 11(1), 56-95. https://doi.org/10.1177/0272431691111004

Baumrind, D. (1991b). Parenting Styles and adolescent development. En J. Brooks- Gunn, R. Lerner \& A. C. Petersen (Eds.), The encyclopedia of adolescence (pp. 746-758). New York: Garland.

Baumrind, D. (1994). The social context of child maltreatment. Family Relations, 43(4), 360-368. https://doi.org/10.2307/585365

Baumrind, D. (1996). The discipline controversy revisited. Family Relations: Journal of Applied Family \& Child Studies, 45(4), 405-414. https://doi.org/10.2307/585170

Behnke, A. O., Plunkett, S. W., Sands, T., \& Bámaca-Colbert, M. Y. (2011). The Relationship Between Latino Adolescents' Perceptions of Discrimination, Neighborhood Risk, and Parenting on Self-Esteem and Depressive Symptoms. Journal of Cross-Cultural Psychology, 42(7), 1179-1197. https: / / doi.org/10.1177/0022022110383424

Benson, M. J., Buehler, C., \& Gerard, J. M. (2008). Interparental hostility and early adolescent problem behavior: Spillover via maternal acceptance, harshness, inconsistency, and intrusiveness. The Journal of Early Adolescence, 28(3), 428- 454. https://doi.org/10.1177/0272431608316602

Brody, G. H., Beach S. R. H., Philibert, R. A., Chen, Y., Lei, M., McBride Murry, V., \& Brown A. C. (2009). Parenting moderates a genetic vulnerability factor in longitudinal increases in youths' substance use. Journal of Consulting and Clinical Psychology, 77(1), 1-11. https://doi.org/10.1037/a0012996

Caycedo, C., Ballesteros, B. P., Novoa, M. M., Garcia, D. R., Arias, A. L., Heyck, L. V., Valero, A. P., \& Vargas, R. (2005). Relación entre Variables de Control Parental y Prácticas de Juego en Niños y Niñas de 10 a 13 Años de Edad en la Ciudad de Bogotá. Revista Latinoamericana de Ciencias Sociales, Niñez y Juventud, 3(1), 2-21.

Cheah, C. S. L., Leung, C. Y. Y. C., Tahseen, M., \& Schultz, D. (2009). Authoritative parenting among immigrant Chinese mothers of preschoolers. Journal of Family Psychology, 23(3), 311-320. https://doi.org/10.1037/a0015076

Chyung, Y. J. \& Lee, J. (2008). Intimate Partner Acceptance, Remembered Parental Acceptance in Childhood, and Psychological Adjustment Among Korean College Students in Ongoing Intimate Relationships. Cross-Cultural Research, 42(1), 77- 86. https://doi.org/10.1177/1069397107309857

Clark, T. T., Belgrave, F. Z., \& Abell, M. (2012). The Mediating and Moderating Effects of Parent and Peer Influences Upon Drug Use Among African American Adolescents. Journal of Black Psychology, 38(1), 52-80. https://doi.org/10.1177/0095798411403617

Darling, N. \& Steinberg, L. (1993). Parenting Style as a Context: An Integrative Model. Psychological Bulletin, 113(3), 487-496. https:// doi.org/10.1037/0033-2909.113.3.487

de la Iglesia, G. (2013). Parentalidad percibida en universitarios: afrontamiento, apoyo social y rendimiento (Tesis de doctorado, Universidad de Buenos Aires). Repositorio Digital Institucional de la Universidad de Buenos Aires. http://biblioteca.psi.uba.ar//cgi-bin/koha/opac-detail.pl?biblionumber=51009 
de la Iglesia, G., Castro Solano, A., \& Fernández Liporace, M. (2016). Evidence of validity of the LSB-50: cross-validation, factorial invariance and external criterion analysis. Ciencias Psicológicas, 10(1), 6373. https://doi.org/10.22235/cp.v10i2.1154

de la Iglesia, G., Fernández Liporace, M., \& Castro Solano, A. (2015). Psychometric study of the main clinical scales of the Listado de Sintomas Breve (LSB-50) -Short Checklist of Symptoms- in Argentinean adolescents. Testing, Psychometrics, Methodology in Applied Psychology, 22(3), 383-399.

de Rivera, L., \& Abuín, M. L. (2012). LSB-50 Listado de Sintomas Breve: Manual. Madrid, Spain: TEA Ediciones.

del Campo, S. (2004). Las transiciones de la familia española, Arbor, 702, 451-464. https://doi.org/10.3989/arbor.2004.i702.575

Dwairy, M. (2008). Parental inconsistency versus parental authoritarianism: association with symptoms of psychological disorders. Journal of Youth and Adolescence, 37, 616-626. https://doi.org/10.1007/s10964-007-9169-3

Dwairy, M. \& Dor, A. (2009). Parenting and psychological adjustment of adolescent immigrants in Israel. Journal of Family Psychology, 23(3), 416-425. https://doi.org/10.1037/a0015830

Erikson, E. H. (1963). Childhood and society. New Cork: Norton.

Farrant, B. M., \& Zubrick, S. R. (2012). Early vocabulary development: The importance of joint attention and parent-child book reading. First Language, 32(3), 343-364. https://doi.org/10.1177/0142723711422626

Garcia, F. \& Gracia, E. (2009). Is always authoritative the optimum parenting style? Evidence from Spanish families. Adolescence, 44(173), 101-131.

Gaylord-Harden, N. K., Campbell, C. L., \& Kesselring, C. M. (2010). Maternal Parenting Behaviors and Coping in African American Children: The Influence of Gender and Stress. Journal of Children and Family Studies, 19, 579-587. https://doi.org/10.1007/s10826-009-9333-3

Jennings, K. D., Sandberg, I., Kelley, S. A., Valdes, L., Yaggi, K., Abrew, A., \& Macey-Kalcevic, M. (2008). Understanding of the self and maternal warmth predict later self-regulation in toddlers. International Journal of Behavioral Development, 32(2), 108-118. https://doi.org/10.1177/0165025407087209

Kaiser, N. M, McBurnett, K., \& Pfiffner, L. J. (2011). Child ADHD Severity and Positive and Negative Parenting as Predictors of Child Social Functioning: Evaluation of Three Theoretical Models. Journal of Attention Disorders, 15(3), 193-203. https://doi.org/10.1177/1087054709356171

Khaleque, A., \& Rohner, R. P. (2012). Transnational relations between perceived parental acceptance and personality dispositions of children and adults: A meta-analytic review. Personality and Social Psychology Review, 16(2), 103-115. https://doi.org/10.1177/1088868311418986

Kasser, T., Koestner, R., \& Lekes, N. (2002). Early Family Experiences and Adult Values: A 26-Year, Prospective Longitudinal Study. Personality and Social Psychology Bulletin, 28(6), 826-835. https://doi.org/10.1177/0146167202289011

Keyes, C. L. M. (2005). Mental illness and/or mental health? Investigating axioms of the complete state model of health. Journal of Consulting and Clinical Psychology, 73, 539-548. https://doi.org/10.1037/0022-006X.73.3.539

Kim, K. \& Rohner, R. P. (2002). Parental warmth, control, and involvement in schooling: Predicting academic achievement among Korean American adolescents. Journal of Cross-Cultural Psychology, 33(2), 127-140. https://doi.org/10.1177/0022022102033002001

Kokkinos, C. M. \& Voulgaridou, I. (2016): Links between relational aggression, parenting and personality among adolescents. European Journal of Developmental Psychology, 14(3), 249-264. https://doi.org/10.1080/17405629.2016.1194265

Lee, J. (2011). The influence of parent- adolescent relationship quality, parental monitoring, and Using peer substance use on substance use in South Korean high school students: latent growth curve modelling. Health Education Journal, 71(4), 505-515. https://doi.org/10.1177/0017896911409733

Lengua, L. J. \& Kovacs, E. A. (2005). Bidirectional associations between temperament and parenting and the prediction of adjustment problems in middle childhood. Journal of Applied Developmental Psychology, 26(1), 21-38. https://doi.org/10.1016/j.appdev.2004.10.001

Lohaus, A., Vierhaus, M., \& Ball, J. (2009). Parenting Styles and Health-Related Behavior in Childhood and Early Adolescence: Results of a Longitudinal Study. The Journal of Early Adolescence, 29(4), 449-475. https://doi.org/10.1177/0272431608322954

López, G. C. H., Palacio, C. R. \& Nieto, L. A. R. (2007). Las prácticas educativas familiares como facilitadores del proceso de desarrollo en el niño y niña. Grupo de estudios clínicos y sociales en Psicología Universidad de San Buenaventura. El Ágora, 7(2), 235-237.

Lorenzo-Seva, U. \& Ferrando, P. J. (2013). FACTOR 9.2: A comprehensive program for fitting exploratory and semiconfirmatory factor analysis and IRT models. Applied Psychological Measurement, 37(6), 497498. https://doi.org/10.1177/0146621613487794

Luecken, L. J., Appelhans, B. M., Kraft, A., \& Brown, A. (2006). Never far from home: A cognitive-affective model of the impact of early-life family relationships on physiological stress responses in adulthood. Journal of Social and Personal Relationships, 23(2), 189-203. https://doi.org/10.1177/0265407506062466

Lupano Perugini, M. L., de la Iglesia, G., Castro Solano, A., \& Keyes, M. C. (2017). The Mental Health Continuum-Short Form (MHC-SF) in the Argentinean Context: Confirmatory Factor Analysis and Measurement Invariance. Europe's Journal of Psychology, 13(1), 93-108. https://doi.org/10.5964/ejop.v13i1.1163

Maccoby, E. E. (1992). The role of parents in the socialization of children: An historical overview. Developmental Psychology, 28(6), 1006-1017. https://doi.org/10.1037/0012-1649.28.6.1006

Maccoby, E. E. (1994). The role of parents in the socialization of children: An historical overview. En R. D. 
Parke, P. A. Ornstein, J. J. Rieser \& C. Zahn-Waxler (Eds.), A century of developmental psychology (pp. 589-615). Washington, DC: American Psychological Association. https://doi.org/10.1037/10155-021

Maccoby, E. E. \& Martin, J. (1983). Socialization in the context of the family: Parent- child interaction. En E. M. Hetherington \& P. H. Mussen (Eds.), Handbook of child psychology: Vol. 4. Socialization, personality, and social development (pp. 1-101). New York: Wiley.

Mehrad Sadr, M. (2016). The role of personality traits and perceived parenting styles in predicting cognitive development. International Journal of Applied Behavioral Sciences, 3(2), 27-34.

Mesurado, B. \& Richaud de Minzi, M. C. (2012). Child's Personality and Perception of Parental Relationship as Correlates of Optimal Experience. Journal of Happiness Studies, 14(1), 199-214. https://doi.org/10.1007/s10902-012-9324-8

Minuchin, S. (1974). Families and Family Therapy. Massachusetts: Harvard University Press.

Missotten, L.C., Luyckx, K., Van Leeuwen, L., Klimstra, T., \& Branje, S. (2016). Adolescents' Conflict Resolution Styles Toward Mothers: The Role of Parenting and Personality. Journal of Child and Family Studies, 25, 2480-2497. https://doi.org/10.1007/s10826-016-0421-x

Moos, R. H. (1993). Coping Responses Inventory Youth Form. Odessa, Florida: Psychological Assessment Resources.

Muljat, A. M., Wilson, B. J., Oliver, T. C., Kottke, S., \& Petaja, H. (2008, agosto). Family expressiveness and child emotionality mediating role of inconsistent parenting. Trabajo presentado en la American Psychological Association 116th Annual Convention, Boston, Massachusetts. https://doi.org/10.1037/e529212008-001

Ocón, J. (2006). Familia adoptiva y cambios en la organización familiar tradicional, Papers, 81, 171-185. https://doi.org/10.5565/rev/papers/v81n0.2037

Ongarato, P., de la Iglesia, G., Stover, J. B., \& Fernández Liporace, M. (2009). Adaptación de un Inventario de Estrategias de Afrontamiento para Adolescentes y Adultos. Anuario de Investigaciones, 16, 383-391.

Ozer, E. J., Flores, E., Tschann, J. M., \& Pasch, L. A. (2011). Parenting Style, Depressive Symptoms, and Substance Use in Mexican American Adolescents. Youth Society, 45(3), 365-388. https://doi.org/10.1177/0044118X11418539

Parker, G., Tupling, H., \& Brown, L.B. (1979) A Parental Bonding Instrument. British Journal of Medical Psychology, 52, 1-10. https://doi.org/10.1111/j.2044-8341.1979.tb02487.x

Pinquart, M. \& Gerke, D. C. (2019). Associations of Parenting Styles with Self-Esteem in Children and Adolescents: A Meta-Analysis. Journal of Child and Family Studies, 28, 2017-2035. https://doi.org/10.1007/s10826-019-01417-5

Reilly, E. E., Stey, P., \& Lapsley, D. K. (2016). A new look at the links between perceived parenting, sociallyprescribed perfectionism, and disordered eating. Personality and Individual Differences, 88, 17-20. https://doi.org/10.1016/j.paid.2015.08.038

Rial Boubeta, A., de la Iglesia, G., Ongarato, P., \& Fernández Liporace, M. (2011). Dimensionalidad del Inventario de Afrontamiento para adolescentes y universitarios. Psicothema, 23(3), 464-474.

Richaud de Minzi, M. C., Lemos, V., \& Mesurado, B. (2011). Relaciones entre la percepción que tienen los niños de los estilos de relación y de la empatía de los padres y la conducta prosocial en la niñez media y tardía. Avances en Psicología Latinoamericana, 29(2), 330-343.

Richaud de Minzi, M. C., Mesurado, B., \& Lemos, V. (2012). Links Between Perception of Parental Actions and Prosocial Behavior in Early Adolescence. Journal of Child and Family Studies, 22, 637-646. https://doi.org/10.1007/s10826-012-9617-x

Richaud de Minzi, M. C. (2006). Loneliness and Depression in Middle and Late Childhood: Its Relationship To Attachment and Parental Styles. Journal of Genetic Psychology, 167(2), 189-210. https://doi.org/10.3200/GNTP.167.2.189-210

Richaud de Minzi, M. C. (2010). Influencia del Modelado de los Padres sobre el Desarrollo del Razonamiento Prosocial en los/las Niños/as. Interamerican Journal of Psychology, 43(1), 187-198.

Riggio, H. R. \& Kwong, W. Y. (2011). Paranoid Thinking, Quality of Relationships With Parents, and Social Outcomes Among Young Adults. Journal of Family Issues, 32(8), 1030-1049. https://doi.org/10.1177/0192513X11403281

Riggio, H. R. (2004). Parental marital conflict and divorce, parent-child relationships, social support, and relationship anxiety in young adulthood. Personal Relationships, 11, 99-114. https://doi.org/10.1111/j.1475-6811.2004.00073.x

Ripoll-Nuñez, K. \& Álvarez, C. (2008). Perceived Intimate Partner Acceptance, Remembered Parental Acceptance, and Psychological Adjustment Among Colombian and Puerto Rican Youth and Adults. Cross-Cultural Research, 42(1), 23-34. https://doi.org/10.1177/1069397107309859

Rohner, R. P. \& Veneziano, R. A. (2001). The importance of father love: history and contemporary evidence. Review of General Psychology, 5(4), 382-405. https://doi.org/10.1037/ 1089-2680.5.4.382

Rohner, R. P. (2004). The parental "acceptance-rejection syndrome": Universal correlates of perceived rejection. American Psychologist, 59, 827-840. https://doi.org/10.1037/0003-066X.59.8.830

Rohner, R. P. (2010). Perceived Teacher Acceptance, Parental Acceptance, and the Adjustment, Achievement, and Behavior of School-Going Youths Internationally. Cross-Cultural Research, 44(3), 211-221. https://doi.org/10.1177/1069397110366849

Rohner, R. P., Khaleque, A., Elias, M. S., \& Sultana, S. (2010). The Relationship Between Perceived Teacher and Parental Acceptance, School Conduct, and the Psychological Adjustment of Bangladeshi Adolescents. Cross-Cultural Research, 44(3) 239-252. https://doi.org/10.1177/1069397110366900

Rohner, R. P., Saavedra J. M., \& Granum, E. O. (1978). Development and validation of the personality assessment questionnaire: Test manual. Ann Arbor, MI: University of Michigan

Rothrauff, T. C., Cooney, T. M. \& An, J. S. (2009). Remembered parenting styles and adjustment in middle and late adulthood. Journal of Gerontology: Social Sciences, 64B(1), 137-146. 
https://doi.org/10.1093/geronb/gbn008

Ruiz Becerril, D. (2004). Nuevas formas familiares. Portularia, 4, 219-230.

Saint-Jacques, M. C. \& Lépine, R. (2009). Le style parental des Beaux-Péres dans les familles recomposées. Canadian Journal of Behavioral Science, 41(1), 22-30. https://doi.org/10.1037/a0013566

Schaefer, E. S. (1965). Children's reports of parental behavior: an inventory. Child Development, 36, 414424. https://doi.org/10.2307/1126465

Schaffer, M., Clark, S., \& Jeglic, E. L. (2009). The Role of Empathy and Parenting Style in the Development of Antisocial Behaviors. Crime \& Delinquency, 55(4), 586-599. https://doi.org/10.1177/0011128708321359

Schwarz, B., Mayer, B., Trommsdorff, G., Ben-Arieh, A., Friedlmeier, M., Lubiewska, K., ... Peltzer, K. (2011). Does the Importance of Parent and Peer Relationships for Adolescents' Life Satisfaction Vary Across Cultures? The Journal of Early Adolescence, 32(1), 55-80. https://doi.org/10.1177/0272431611419508

Serot, N. M. \& Teevan R. C. (1961). Perception of the parent-child relationship and its relation to child adjustment. Child Development, 32, 373-378. https://doi.org/10.2307/1125951

Sessa, F. M., Avenevoli, S., Steinberg, L., \& Morris, A. S. (2001). Correspondance among informants on parenting: preschool children, mothers and observers. Journal of Family Psychology, 15(1), 53-68. https://doi.org/10.1037/0893-3200.15.1.53

Simons, L. G. \& Conger, R. D. (2007). Linking mother-father differences in parenting to a typology of family parenting styles and adolescent outcomes. Journal of Family Issues, 28(2), 212-241. https://doi.org/10.1177/0192513X06294593

Sun, Q. W., Ng, K. M., \& Guo, L. (2010). The Link Between Parental Bonding and Adult Attachment in Chinese Graduate Students: Gender Differences. The Family Journal, 18(4), 386-394. https://doi.org/10.1177/1066480710372920

Timmerman, M. E. \& Lorenzo-Seva, U. (2011). Dimensionality assessment of ordered polytomous items with parallel analysis. Psychological Methods, 16, 209-220. https://doi.org/10.1037/a0023353

Valiente, C., Lemery-Chalfant, K., \& Swanson, J. (2009). Children's responses to daily social stressors: Relations with parenting, children's effortful control, and adjustment. Journal of Child Psychology and Psychiatry, 50, 707-717. https://doi.org/10.1111/j.1469-7610.2008.02019.x

Xu, Y., Lin, L., Yang, L., Zhou, L., Tao, Y., Chen, W., ... Wang, W. (2016). Personality disorder and perceived parenting in Chinese students of divorced and intact families. The Family Journal, 24(1), 70-76. https://doi.org/10.1177/1066480715618652

Zhou, Q., Wang, Y., Deng, X., Eisenberg, N., Wolchik, S. A., \& Tein, J. T. (2008). Relations of parenting and temperament to Chinese children's experience of negative life events, coping efficacy, and externalizing problems. Child Development, 79, 493-513. https://doi.org/10.1111/j.1467-8624.2008.01139.x

Recibido 02-06-2020 | Aceptado 14-08-2020

Este trabajo se encuentra bajo una Licencia Creative Commons Atribución 4.0 Internacional que permite a terceros utilizar lo publicado siempre que se dé el crédito pertinente a los autores y a Psicodebate 